\title{
Decreased activity of daily living produced by the combination of Alzheimer's disease and lower limb fracture in elderly requiring nursing care
}

\author{
Toshimitsu Inagawa - Toshio Hamagishi - Yuji Takaso - Yoshiaki Hitomi - Yasuhiro Kambayashi • \\ Yuri Hibino - Aki Shibata • Nguyen T. M. Ngoc · Jiro Okochi - Kotaro Hatta • \\ Kiyoshi Takamuku $\cdot$ Tadashi Konoshita $\cdot$ Hiroyuki Nakamura
}

Received: 11 March 2012/Accepted: 18 April 2012/Published online: 11 May 2012

(C) The Japanese Society for Hygiene 2012

\begin{abstract}
Objectives Alzheimer's disease (AD) impairs cognitive functions, subsequently decreasing activity of daily living (ADL), and is frequently accompanied by lower limb fracture including hip fracture in the elderly. However, there have been few studies on what kinds of physical functions are affected or what degrees of dysfunction are produced by this combination. This study aims to clarify the relationship between decreased ADL and the combination of $\mathrm{AD}$ and lower limb fracture.

Methods We examined present illness and ADL in 4340 elderly aged $82.8 \pm 9.36$ years [average \pm standard deviation (SD)] requiring nursing care and compared ADL between elderly with and without AD or lower limb fracture treated with surgery or conservatively using analysis of covariance (ANCOVA), with age and sex as covariants. Results We recognized that activities of cognitive function $(p<0.001)$, eating (dysphagia) $(p<0.001)$, eating (feeding) $(p<0.001)$, and toilet use $(p<0.001)$ in the elderly with $\mathrm{AD}$ were significantly lower than in those without the disease, even after adjusting for sex and age. Activities of bed mobility $(p<0.05)$, transfer and locomotion $(p<0.001)$, and bathing $(p<0.05)$ in the elderly with a fracture treated with surgery were significantly lower, which differed from the results of AD. Significant interactions of AD and fracture treated with surgery on the ADL scores for bed mobility ( $p<0.001)$, dysphagia $(p<0.01)$, feeding $(p<0.001)$, and
\end{abstract}

T. Inagawa $\cdot$ T. Hamagishi · Y. Takaso - Y. Hitomi ·

Y. Kambayashi · Y. Hibino - A. Shibata ·

N. T. M. Ngoc $\cdot$ J. Okochi $\cdot$ K. Hatta $\cdot$ K. Takamuku ·

T. Konoshita $\cdot$ H. Nakamura $(\bowtie)$

Graduate School of Medical Science,

Kanazawa University, Kanazawa 920-8640, Japan

e-mail: hiro-n@po.incl.ne.jp toilet use $(p<0.05)$ show that the combination had a much more profound influence on the ADL scores than AD or fracture alone. We obtained almost the same results for fractures treated conservatively as for fractures treated with surgery.

Conclusions These results demonstrated that the combined effects of $A D$ and lower limb fracture were significantly greater than expected additive effects of $\mathrm{AD}$ and fracture, suggesting that the combination of $\mathrm{AD}$ and lower limb fracture has synergistic effects on almost all types of ADL except cognitive functions.

Keywords Alzheimer's disease - Hip fracture . Elderly $\cdot$ Activity of daily living $\cdot$ Physical function

\section{Introduction}

Alzheimer's disease (AD) is the most common form of dementia. The number of individuals with $\mathrm{AD}$ has been increasing considerably in recent years, accounting for more than $50 \%$ of dementia cases [1]. AD causes cognitive impairment of elderly in need of care, consequently interfering with daily tasks and decreasing activities of daily living (ADL) in many ways [2]. It is known that patients with $\mathrm{AD}$ suffer from various kinds of complications [3]. The increased risk of falling associated with $A D$ leads to lower limb fracture including hip fracture [4]. In addition, taken together with the fact that $\mathrm{AD}$ and osteoporosis have common risk factors $[5,6]$, the incidence of fracture is higher in elderly with $\mathrm{AD}$. Thus, lower limb fractures could accompany $\mathrm{AD}$ as one of its complications.

To make matters worse, elderly made bedridden by the aftereffects of lower limb fracture could develop cognitive impairment as its complication [7]. Even if an elderly person 
is not made bedridden, the aftereffects of fracture sometimes impair cognitive functions, subsequently decreasing basic ADL [8-10]. AD and fracture, which are commonly encountered in the elderly, aggravate each other, being the two major factors subsequently deteriorating ADL in bedridden elderly. Therefore, $\mathrm{AD}$ and fracture produce a vicious spiral, resulting in not only cognitive impairments but also deterioration in ADL $[3,5]$. However, it remains to be elucidated exactly what kinds of physical functions are affected and to what degree ADL is changed by the combination of $\mathrm{AD}$ and lower limb fracture. To clarify this, we performed a national survey in nursing care institutions in Japan, examining the decreased ADL in elderly with and without AD and lower limb fracture.

\section{Subjects and methods}

\section{Subjects}

Three hundred and ninety facilities were randomly selected out of 3410 nursing care institutions in Japan. Ten resident patients and 10 daycare patients were sampled at random from each facility. A total of 7800 patients were prospective subjects in this study. We mailed a questionnaire to caregivers in each facility inquiring about ADL and medical conditions, including $\mathrm{AD}$ and fracture, as described below and obtained 4340 responses $(55.6 \%, 2132$ resident patients and 2208 daycare patients). This survey was conducted from December 2009 to February 2010. The average age and SD of the 4340 subjects were 82.8 and 9.36 years. This research was conducted after obtaining approval from the ethical committee of the Japan National Conference of Geriatric Health Care Facility.

\section{Questionnaire}

The questionnaires regarding medical conditions in subjects were related to $\mathrm{AD}$ and lower limb fracture (hip fracture and lower leg fracture) requiring surgery, and fractures treated without surgery. To examine AD, the questions concerned neuropsychological examinations including the patients' medical history, neurological testing, Mini-Mental State Examination [11], and standard clinical evaluation including brain scanning. In the diagnostic process, DSM-III [12] was used for diagnosis and to rule out other factors. The National Institute of Neurological and Communicative Disorders and Stroke-Alzheimer's Disease and Related Disorders Association guidelines [13] were used for detection and diagnosis of either possible or probable AD. Lower limb fracture was diagnosed after an appropriate procedure including X-ray roentgenogram. The diagnoses of $\mathrm{AD}$ and fracture were performed by a physician of the facility within 1 year prior to the contact with nursing care institutions for elderly in this study. Our subjects included 491 patients with possible or probable AD, 405 and 196 patients with fracture treated with surgery and conservatively, respectively, and 3248 elderly without AD or fracture.

Regarding ADL, the questionnaire was developed on the basis of a standard described previously [14, 15]. They dealt with bed mobility, transfer and locomotion, cognitive function (orientation), cognitive function (communication), cognitive function (mental activity), eating (dysphagia), eating (feeding), toilet use, and bathing for elderly requiring both residential nursing care and daycare. Each ADL was categorized into a five-point scale. In the case of bed mobility, for example, a score of 5 represents being able to stand on one's feet and maintain this posture; score 4: having difficulty maintaining a standing posture, but being able to transfer from one place to another in a sitting position; score 3 : being unable to move in a sitting position, but being able to sit in a proper posture without support; score 2: having difficulty sitting in an upright posture, but being able to turn over on a bed; and score 1: not being able to turn over on a bed. In the case of eating (feeding), a score of 5 represents being able to eat well without any support of others; score 4: spilling food during eating; score 3 : having difficulty eating by themselves, but being able to eat with support for their posture and position of the dish; score 2: being unable to eat without complete support; and score 1: being unable to eat with any support (receiving tube feeding). Thus, the requirement for more concentrated nursing care during eating for the elderly decreased the ADL score. A lower score for each ADL implies worse ADL. In Table 1, we show ADL and prevalence of $\mathrm{AD}$, fracture treated with surgery, and fracture treated conservatively according to the status of nursing care (residential and daycare).

\section{Statistics}

Pearson's correlation coefficients of age showed weak but significant correlations with bed mobility $(-0.118)$, transfer and locomotion $(-0.145)$, cognitive function (orientation) (-0.262), cognitive function (communication) $(-0.199)$, cognitive function (mental activity) $(-0.232)$, eating (dysphagia) (-0.142), eating (feeding) $(-0.088)$, toilet use $(-0.126)$, and bathing $(-0.077)$ (all $p<0.001)$. Because of significant differences in proportions in terms of sex and age between elderly with and without diseases in addition to significant correlations between age and ADL scores, we compared each ADL score by analysis of covariance (ANCOVA) with age and sex as covariants, in which age was assigned as a continuous variable and sex was dummy-coded as follows: male 0 , female 1 . Mean 


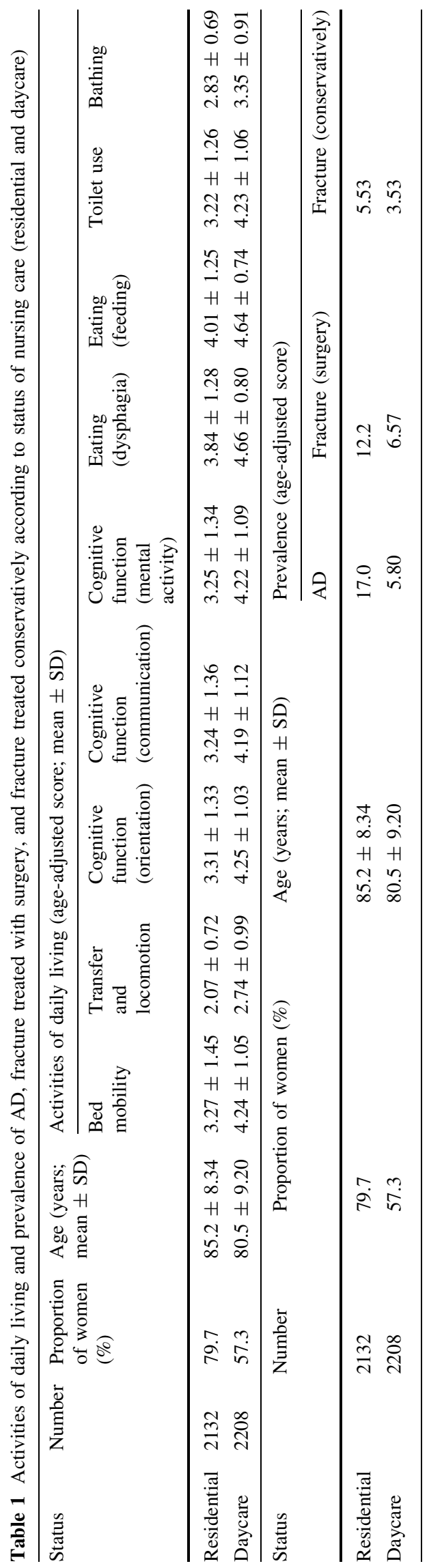

ADL score and standard deviation adjusted by age and sex were calculated using regression coefficients corresponding to age, sex, and disease obtained by ANCOVA and raw mean values of age and sex in total subjects. To assess the interaction for $\mathrm{ADL}$ score between the combination of $\mathrm{AD}$ and fracture treated with or without surgery, we used two-way ANCOVA with age and sex as covariants. The statistical software SPSS version 17 was used. Twotailed $p$-values less than $5 \%$ were considered statistically significant.

\section{Results}

Changes in ADL with $\mathrm{AD}$ and fracture

The proportion of women and age of subjects with AD were significantly higher than those without AD. The activities of cognitive function (orientation), cognitive function (communication), cognitive function (mental activity), eating (dysphagia), eating (feeding), and toilet use in the elderly with AD were significantly lower than those without the disease, even after controlling for sex and age (Table 2). Activities of bed mobility, transfer and locomotion, and bathing in elderly with a fracture requiring surgical operation were significantly lower, which were quite different results from AD (Table 3). Table 4 also shows lower scores on bed mobility, transfer and locomotion, and bathing of the patients with a fracture treated without surgery.

The interaction between $\mathrm{AD}$ and fracture

The two-way ANCOVA demonstrated a significant main effect of $\mathrm{AD}$ on ADL scores of all cognitive functions and eating functions, as well as toilet use, and a significant main effect of fracture on all scores of ADL except cognitive functions. Furthermore, we recognized significant interactions between $\mathrm{AD}$ and fracture on the activities of bed mobility, eating (dysphagia), eating (feeding), and toilet use after adjustment for age and sex (Table 5). Table 6 shows ADL scores in the cases of patients with complications of $\mathrm{AD}$ and fracture treated conservatively. ADL scores of all cognitive functions and eating functions, as well as toilet use, in the elderly with $\mathrm{AD}$ were significantly decreased compared with those without AD. The scores of all ADL except cognitive functions and eating functions were significantly decreased compared with those without fracture treated conservatively. Interactions between ADL and fracture treated conservatively were recognized for the scores of bed mobility, transfer and locomotion, and toilet use even after adjusting for sex and age. There were decreases in the scores of eating (dysphagia), eating 

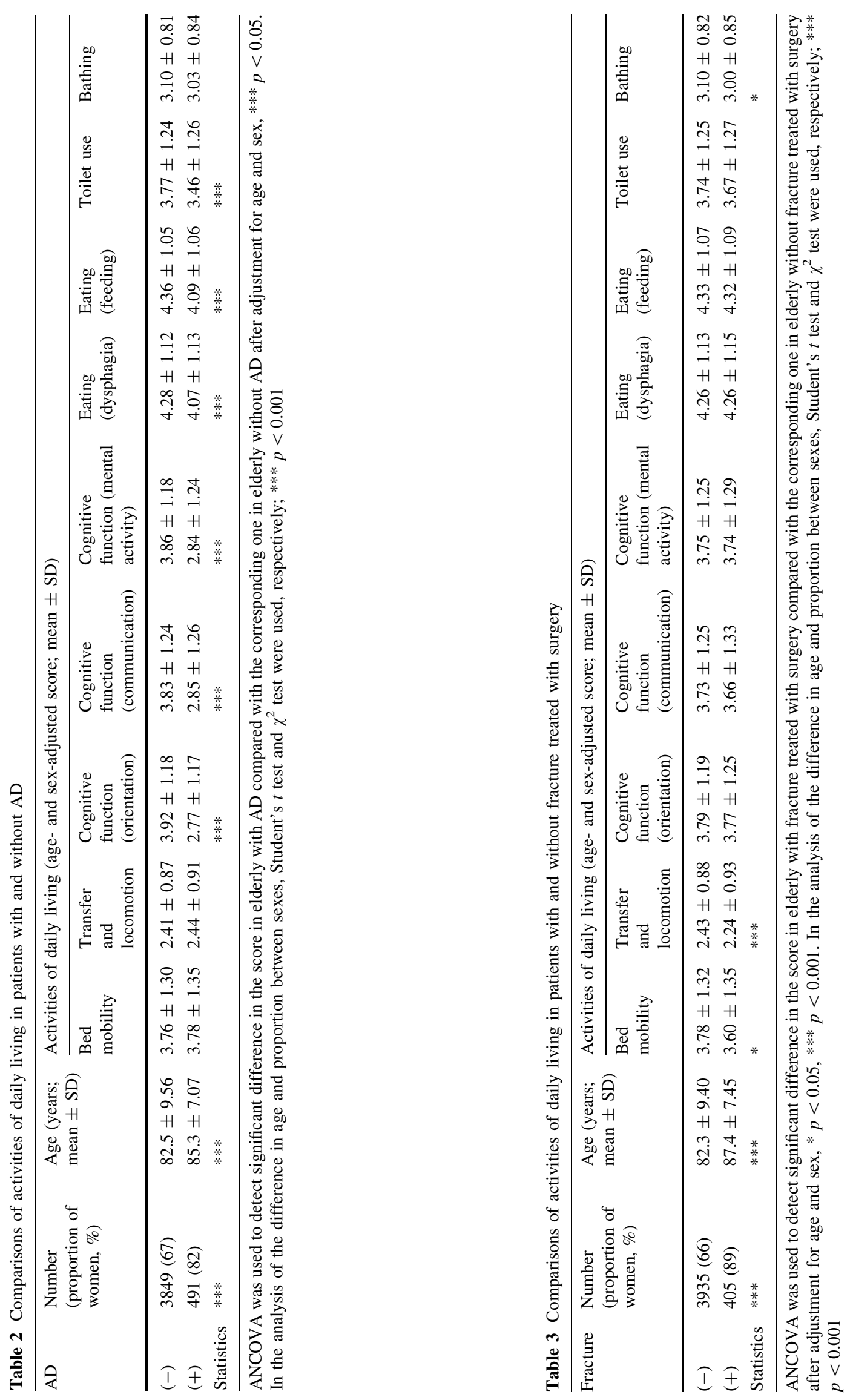

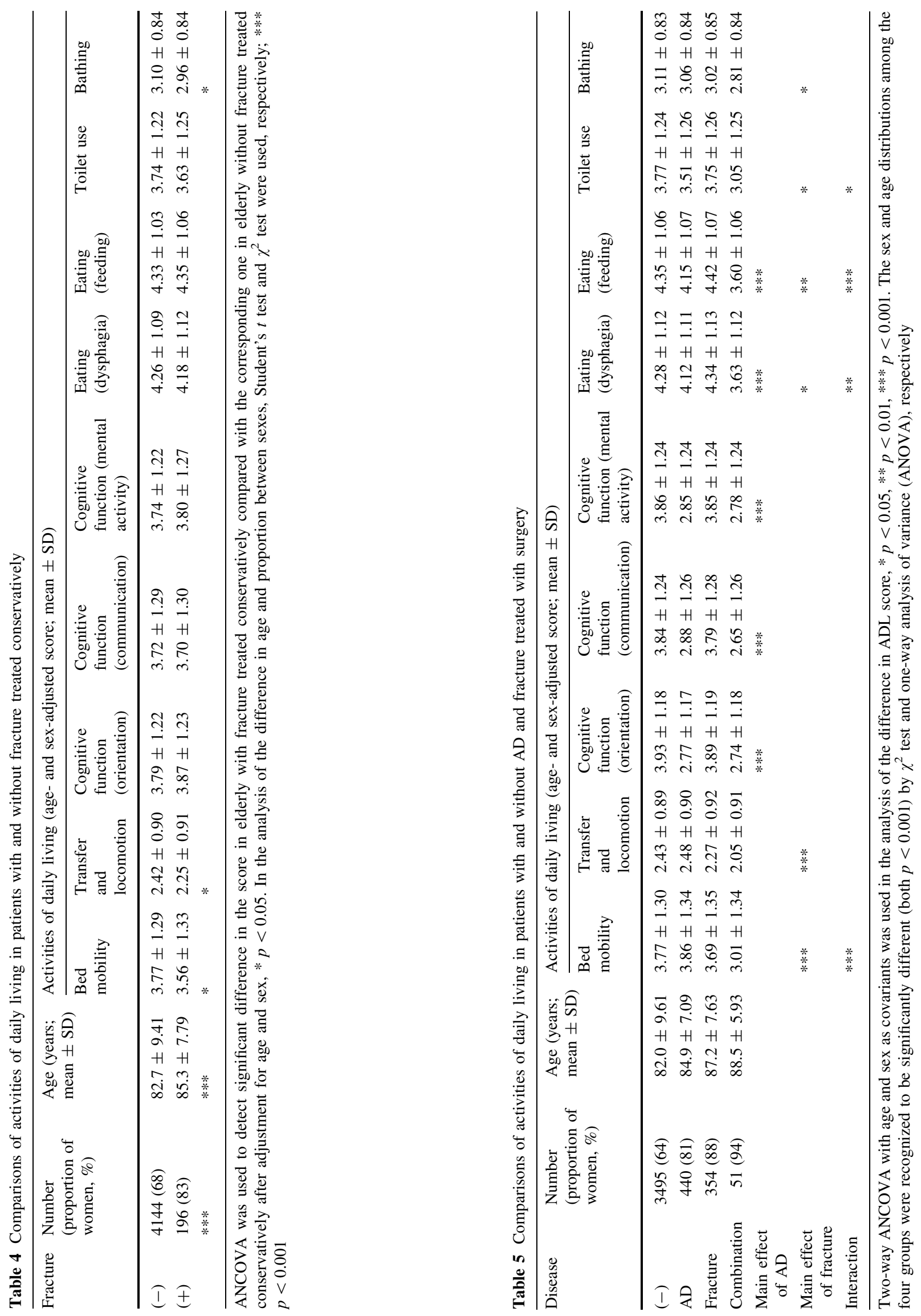
(feeding), and bathing in the patients with the combination, but these were not statistically significant (Table 6).

\section{Discussion}

Decreased ADL in eating (dysphagia), eating (feeding), and toilet use as well as cognitive functions, but not in bed mobility, or transfer and locomotion, were recognized in $\mathrm{AD}$ patients in this study. Although $\mathrm{AD}$ in our subjects was not considered to produce symptoms severe enough to affect motor functions, the influences of $\mathrm{AD}$ on eating (dysphagia), eating (feeding) [16, 17], and toilet use [18] seemed to be associated with cognitive impairments due to AD. These findings are supported by epidemiological studies on the natural course of the progress of AD [19]. In contrast to the results for $\mathrm{AD}$, the patients with fracture treated either with or without surgery showed decreased ADL scores in bed mobility, transfer and locomotion, and bathing. Motor dysfunctions including difficulties in bathing are common following a hip fracture or a lower limb fracture [20, 21]. Thus, many epidemiological studies have evaluated effects of $\mathrm{AD}$ or lower limb fracture on individual ADL in the elderly, albeit separately.

The kinds of function affected and the degrees of change in ADL in patients suffering from the combination of $\mathrm{AD}$ and lower limb fracture are clarified by the observation that the kinds of dysfunction for $\mathrm{AD}$ and fracture were quite different. Our results showed significant interactions on the ADL scores of bed mobility, eating (dysphagia), eating (feeding), and toilet use, demonstrating that the combination of $\mathrm{AD}$ and fracture treated with surgery has more profound influences on the ADL scores than $\mathrm{AD}$ or fracture alone. This implies that the combined effect was significantly greater than the expected additive effect of $\mathrm{AD}$ and fracture. The decreased ADL score of transfer and locomotion with this combination was not recognized as involving a significant interaction between $\mathrm{AD}$ and fracture with surgery, but it was in cases without surgery. Thus, the combined effects of $\mathrm{AD}$ and lower limb fracture on ADL excluding cognitive functions and bathing were much larger than the sum of the sole effects of $\mathrm{AD}$ and fracture. The lack of an interaction for cognitive functions seems to be due to severe influences of $\mathrm{AD}$ on cognitive functions, for which complication with fracture did not bring any further deterioration $[2,3]$.

A fracture in a lower limb has a much greater influence on toilet use because this ability largely depends upon the functions of the lower limbs [18]. However, there are few studies demonstrating how the complication of lower limb fracture with $\mathrm{AD}$ affects dysphagia or eating activity, which is unaffected by the fracture alone [22]. Our findings 
showing that fracture in a lower limb aggravates dysfunctions in eating (dysphagia) and eating (feeding) produced by $\mathrm{AD}$ suggest that the fracture makes $\mathrm{AD}$ worse and more progressive. This finding is supported by many studies [2325], which have shown that a lower limb fracture affects the central nervous system through motor dysfunction, resulting in cognitive impairment.

On the other hand, our finding showing that the activities of bed mobility, and transfer and locomotion that were decreased by the fracture were further deteriorated by its complication with $\mathrm{AD}$ supports the idea that $\mathrm{AD}$ has greater influence on motor functions than expected. It is well known that osteoporosis, which often accompanies $\mathrm{AD}$, leads to motor dysfunctions [5]. Some studies have demonstrated that problems in abnormal behaviors in $\mathrm{AD}$ patients may produce muscular dystrophy, which further damages physical functions $[18,25]$. Taking these findings together, motor dysfunctions such as in bed mobility, and transfer and locomotion are considered to be accelerated by the complication of fracture with AD.

Women are at higher risk of developing $\mathrm{AD}$ and fracture $[26,27]$. Our results showed that women account for $94 \%$ of patients with the complications of $\mathrm{AD}$ and fracture. Therefore, the synergistic effects of AD and lower limb fracture on ADL seen in this study explain well that ADL in all fields in elderly women are lower than those in elderly men of the same age.

There were few differences in decreased ADL between cases of fracture treated with and without surgery. Our data regarding fracture treated with and without surgery do not coincide well with the findings that patients with fracture treated with surgery show better prognosis in terms of ADL than those treated without surgery $[7,10]$. We did not obtain any data regarding social circumstances and physical background under which the patients with fracture did not receive surgery. Analysis of the data might confirm effects of surgery on the recovery from fracture, which was not seen in this study. Since this study was performed with a cross-sectional design, the causal effects of $\mathrm{AD}$ and fracture on ADL must be demonstrated by further follow-up study.

In conclusion, our results showed that the combination of $\mathrm{AD}$ and lower limb fracture has more profound influence on ADL scores in terms of bed mobility, transfer and locomotion, eating (dysphagia), eating (feeding), and toilet use, demonstrating that the combined effects were significantly greater than expected additive effects of $\mathrm{AD}$ and fracture, suggesting that the complication of $\mathrm{AD}$ with lower limb fracture has synergistic effects on almost all types of ADL besides cognitive functions. The interaction between $\mathrm{AD}$ and fracture on ADL seemed to be due to effects of both $\mathrm{AD}$ on motor function and fracture on central nervous functions.
Acknowledgments The work was funded by a Grant-in-Aid for Scientific Research (B) from the Ministry of Health, Labor, and Welfare (2009-2010), Japan.

Conflict of interest None.

\section{References}

1. Reitz C, Brayne C, Mayeux R. Epidemiology of Alzheimer disease. Nat Rev Neurol. 2011;7(3):137-52.

2. Riccio D, Solinas A, Astara G, Mantovani G. Comprehensive geriatric assessment in female elderly patients with Alzheimer disease and other types of dementia. Arch Gerontol Geriatr. 2007; 44(Suppl 1):343-53.

3. Malone DC, McLaughlin TP, Wahl PM, Leibman C, Arrighi HM, Cziraky MJ, et al. Burden of Alzheimer's disease and association with negative health outcomes. Am J Manag Care. 2009;15(8): 481-8.

4. Mazoteras Munoz V, Abellan van Kan G, Cantet C, Cortes F, Ousset PJ, Rolland Y, et al. Gait and balance impairments in Alzheimer disease patients. Alzheimer Dis Assoc Disord. 2010; 24(1):79-84.

5. Tysiewicz-Dudek M, Pietraszkiewicz F, Drozdzowska B. Alzheimer's disease and osteoporosis: common risk factors or one condition predisposing to the other? Ortop Traumatol Rehabil. 2008;10(4):315-23.

6. Zhou R, Deng J, Zhang M, Zhou HD, Wang YJ. Association between bone mineral density and the risk of Alzheimer's disease. J Alzheimers Dis. 2011;24(1):101-8.

7. Pretto M, Spirig R, Kaelin R, Muri-John V, Kressig RW, Suhm $\mathrm{N}$. Outcomes of elderly hip fracture patients in the Swiss healthcare system: a survey prior to the implementation of DRGs and prior to the implementation of a geriatric fracture Centre. Swiss Med Wkly. 2010;140:w13086.

8. Givens JL, Sanft TB, Marcantonio ER. Functional recovery after hip fracture: the combined effects of depressive symptoms, cognitive impairment, and delirium. J Am Geriatr Soc. 2008;56(6):1075-9.

9. Ekström W, Miedel R, Ponzer S, Hedström M, Samnegård E, Tidermark J. Quality of life after a stable trochanteric fracture-a prospective cohort study on 148 patients. J Orthop Trauma. 2009; 23(1):39-44.

10. Nielsen KA, Jensen NC, Jensen CM, Thomsen M, Pedersen L, Johnsen SP, et al. Quality of care and 30 day mortality among patients with hip fractures: a nationwide cohort study. BMC Health Serv Res. 2009;9:186.

11. Folstein MF, Folstein SE, McHugh PR. "Mini-mental state". A practical method for grading the cognitive state of patients for the clinician. J Psychiatr Res. 1975;12(3):189-98.

12. American PA. Diagnostic and statistical manual of mental disorders. Revised 3rd ed. Washington; 1987.

13. McKhann G, Drachman D, Folstein M, Katzman R, Price D, Stadlan EM. Clinical diagnosis of Alzheimer's disease: report of the NINCDS-ADRDA Work Group under the auspices of Department of Health and Human Services Task Force on Alzheimer's Disease. Neurology. 1984;34(7):939-44.

14. Hughes LF, Perkins K, Wright BD, Westrick H. Using a Rasch scale to characterize the clinical features of patients with a clinical diagnosis of uncertain, probable, or possible Alzheimer disease at intake. J Alzheimers Dis. 2003;5(5):367-73.

15. Okochi J, Utsunomiya S, Takahashi T. Health measurement using the ICF: test-retest reliability study of ICF codes and qualifiers in geriatric care. Health Qual Life Outcomes. 2005;3:46. 
16. Correia Sde M, Morillo LS, Jacob Filho W, Mansur LL. Swallowing in moderate and severe phases of Alzheimer's disease. Arq Neuropsiquiatr. 2010;68(6):855-61.

17. Suh MK, Kim H, Na DL. Dysphagia in patients with dementia: Alzheimer versus vascular. Alzheimer Dis Assoc Disord. 2009; 23(2):178-84.

18. Carpenter GI, Hastie CL, Morris JN, Fries BE, Ankri J. Measuring change in activities of daily living in nursing home residents with moderate to severe cognitive impairment. BMC Geriatr. 2006; 6(7):1-8.

19. Zanetti O, Frisoni GB, Rozzini L, Bianchetti A, Trabucchi M. Validity of direct assessment of functional status as a tool for measuring Alzheimer's disease severity. Age Ageing. 1998;27(5):615-22.

20. Penrod JD, Litke A, Hawkes WG, Magaziner J, Koval KJ, Doucette JT, et al. Heterogeneity in hip fracture patients: age, functional status, and comorbidity. J Am Geriatr Soc. 2007;55(3): 407-13.

21. Alarcón T, González-Montalvo JI, Gotor P, Madero R, Otero A. Activities of daily living after hip fracture: profile and rate of recovery during 2 years of follow-up. Osteoporos Int. 2010;22(5): $1609-13$.
22. Mariani E, Monastero R, Mecocci P. Mild cognitive impairment: a systematic review. J Alzheimers Dis. 2007;12(1):23-35.

23. Olofsson B, Lundström M, Borssén B, Nyberg L, Gustafson Y. Delirium is associated with poor rehabilitation outcome in elderly patients treated for femoral neck fractures. Scand J Caring Sci. 2005;19(2):119-27.

24. Moncada LV, Andersen RE, Franckowiak SC, Christmas C. The impact of cognitive impairment on short-term outcomes of hip fracture patients. Arch Gerontol Geriatr. 2006;43(1):45-52.

25. Furlaneto ME, Garcez-Leme LE. Impact of delirium on mortality and cognitive and functional performance among elderly people with femoral fractures. Clinics. 2007;62(5):545-52.

26. Hebert LE, Scherr PA, Bienias JL, Bennett DA, Evans DA. Alzheimer disease in the US population: prevalence estimates using the 2000 census. Arch Neurol. 2003;60(8):1119-22.

27. Samuelsson B, Hedström MI, Ponzer S, Söderqvist A, Samnegård E, Thorngren KG, et al. Gender differences and cognitive aspects on functional outcome after hip fracture-a 2 years' follow-up of 2,134 patients. Age Ageing. 2009;38(6):686-92. 